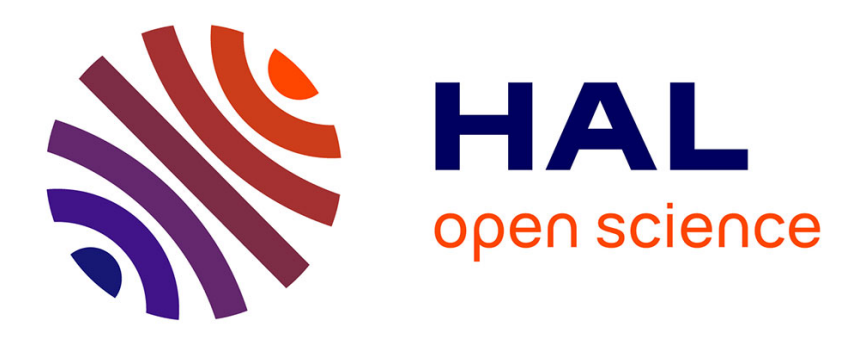

\title{
Les enjeux théoriques du droit de vote des étrangers: la démocratie contre la souveraineté
}

Hervé Andres

\section{To cite this version:}

Hervé Andres. Les enjeux théoriques du droit de vote des étrangers: la démocratie contre la souveraineté. Migrations Société, 2007, 19 (114), pp.47-64. halshs-00189683v2

\section{HAL Id: halshs-00189683 \\ https://shs.hal.science/halshs-00189683v2}

Submitted on 19 Jun 2017

HAL is a multi-disciplinary open access archive for the deposit and dissemination of scientific research documents, whether they are published or not. The documents may come from teaching and research institutions in France or abroad, or from public or private research centers.
L'archive ouverte pluridisciplinaire HAL, est destinée au dépôt et à la diffusion de documents scientifiques de niveau recherche, publiés ou non, émanant des établissements d'enseignement et de recherche français ou étrangers, des laboratoires publics ou privés. 


\section{LES ENJEUX THÉORIQUES DU DROIT DE VOTE DES ÉTRANGERS : LA DÉMOCRATIE CONTRE LA SOUVERAINETÉ}

Hervé Andrès

Centre d'information et d'études sur les migrations internationales | "Migrations Société »

2007/6 N 114 | pages 47 à 64

ISSN 0995-7367

Article disponible en ligne à l'adresse :

http://www.cairn.info/revue-migrations-societe-2007-6-page-47.htm

\section{Pour citer cet article :}

Hervé Andrès, « Les enjeux théoriques du droit de vote des étrangers : la démocratie contre la souveraineté », Migrations Société 2007/6 ( $\left.\mathrm{N}^{\circ} 114\right)$, p. 47-64.

Distribution électronique Cairn.info pour Centre d'information et d'études sur les migrations internationales.

(C) Centre d'information et d'études sur les migrations internationales. Tous droits réservés pour tous pays.

La reproduction ou représentation de cet article, notamment par photocopie, n'est autorisée que dans les limites des conditions générales d'utilisation du site ou, le cas échéant, des conditions générales de la licence souscrite par votre établissement. Toute autre reproduction ou représentation, en tout ou partie, sous quelque forme et de quelque manière que ce soit, est interdite sauf accord préalable et écrit de l'éditeur, en dehors des cas prévus par la législation en vigueur en France. Il est précisé que son stockage dans une base de données est également interdit. 


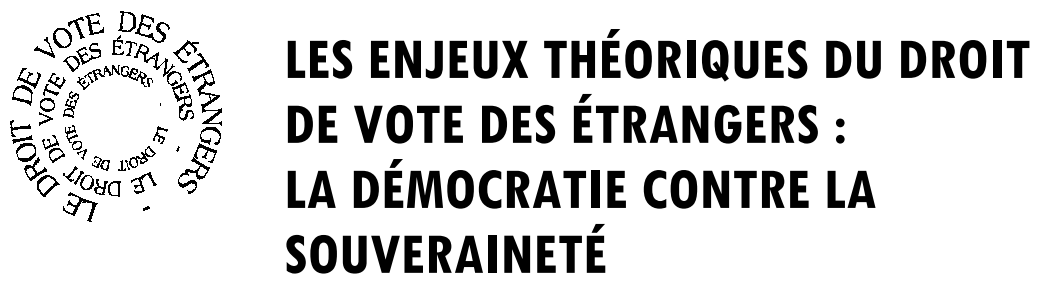

Hervé ANDRÈS *

Il est très opportun de traiter avec attention de la question du droit de vote des étrangers, car elle met en jeu, du point de vue formel, les migrants - voire leurs descendants - en tant que personnes, en tant que phénomène social et en tant que représentations collectives. Mais aussi et surtout, cette question met en jeu la société tout entière dans laquelle elle se pose. Accorder ou non le droit de vote à des étrangers a ainsi un effet miroir : c'est une manière de décrire la société d'accueil que de se consacrer à l'étude du traitement politique que celle-ci réserve aux étrangers. Bien sûr, ce traitement ne se limite aucunement à la seule question du droit de vote, et concerne plus largement et plus directement les questions du droit d'entrée et du droit de séjour, du droit de vivre en famille, du droit du travail, des droits sociaux en général. Ceci dit, les droits politiques (et parmi ceux-ci, le droit de vote) constituent indéniablement un élément important du statut des individus dans une société, et l'on peut même considérer qu'ils participent au socle de tous les autres droits, au " droit d'avoir des droits $\|^{1}$.

L'objectif de la présente contribution est de présenter une interprétation des enjeux théoriques fondamentaux du droit de vote des étrangers $^{2}$. Contrairement aux approches les plus répandues, il ne s'agit pas de se demander s'il faut, ou non, accorder le droit de vote aux étrangers. Il ne s'agit pas de peser le pour et le contre de cette proposition, d'en présenter les arguments favorables et défavorables. II

* Ingénieur d'études, docteur en science politique, Université de Nice, Unité de recherches Migrations et société (URMIS), CNRS, UMR 7032. E-mail : herve.andres@unice.fr

1. ARENDT, Hannah, Les origines du totalitarisme. L'Impérialisme, Paris : Éd. Fayard, 1984, 348 p.

2. Cette contribution synthétise et réordonne une réflexion menée dans le cadre d'une thèse de science politique : ANDRÈS, Hervé, Le droit de vote des étrangers : état des lieux et fondements théoriques, thèse de doctorat de sciences juridiques et politiques, spécialité de philosophie politique, sous la direction de $\mathrm{M}^{\mathrm{me}}$ Chemillier-Gendreau, Université Paris VII-Denis Diderot, École doctorale 382 - EESC - Économie, Espaces, Sociétés, Civilisations : Pensée critique, politique et pratiques sociales, 2007, consultable en ligne à l'adresse http://tel.archives-ouvertes.fr/tel-00130445. 
ne s'agit pas de se demander si une telle proposition est socialement intéressante, juridiquement correcte ou politiquement possible. II ne s'agit pas de discuter des éventuelles restrictions d'un tel droit de vote (à tel ou tel scrutin plus ou moins local, au bout de telle ou telle durée de résidence....). II s'agit, d'une part, principalement à partir des travaux de Jacques Rancière, de montrer que la démocratie (conçue de façon extensive et intensive, et non simplement procédurale) est l'enjeu fondamental de la question du droit de vote des étrangers. Et il s'agit, d'autre part, de montrer comment cette question constitue un point nodal de contradiction dans les fondements du régime entre nationalité et citoyenneté ou entre souveraineté et démocratie.

\section{La démocratie, enjeu fondamental du droit de vote des étrangers}

La relation entre la proposition du droit de vote des étrangers et la démocratie n'a rien d'évident. Le droit de vote ne peut assurément pas être considéré comme l'alpha et l'omega de la démocratie, et pour beaucoup la revendication du droit de vote des étrangers, réitérée en France durant plusieurs décennies, apparaît avec le temps comme une vieille promesse trahie, comme une proposition instrumentalisée par les enjeux politiciens. L'engagement pour une citoyenneté plus active et pour une démocratie plus participative peut faire apparaître comme saugrenue, voire contradictoire, la revendication d'une extension d'un droit de vote jugé limité et trompeur. Pourtant, si l'on part d'une conception quelque peu exigeante de la démocratie, la lutte pour l'universalisation du suffrage et, aujourd'hui, pour le droit de vote des étrangers, participe pleinement à la perspective démocratique, et c'est même la démocratie qui constitue l'enjeu central de la question. La lutte pour l'égalité peut être comprise comme un processus de subjectivation, ayant du sens pour toute la communauté politique.

\section{- Une conception substantielle de la démocratie, basée sur le conflit}

On ne saurait réduire la démocratie à "I une forme de régime représentatif doté présentement du monopole de la légitimité politique ${ }^{3}{ }^{3}$, valorisée depuis Tocqueville et repérable aujourd'hui par quelques

3. BRAUD, Philippe, "Démocratie", in : HERMET, Guy et al. (sous la direction de), Dictionnaire de la science politique et des institutions politiques, Paris : Éd. Armand Colin, 2000, pp. 75-79. 
attributs institutionnels : pluralisme politique, tenue régulière d'élections libres et suffrage universel (et libéralisme économique). Cette vision trop aseptisée est insuffisante et procède d'une réduction apolitique ${ }^{4}$. La démocratie ne saurait être réduite à l'État de droit, et on peut même affirmer que ce n'est vraiment que contre l'État qu'elle peut réellement trouver sa vérité ${ }^{5}$.

De nombreux auteurs récusent la conception institutionnelle ou procédurale de la démocratie ${ }^{6}$ et mettent en évidence que c'est à partir du conflit ${ }^{7}$ qu'on peut véritablement penser la démocratie. C'est à cette conception, qui a pour horizon la liberté, que l'on se rallie dans la présente contribution. La démocratie n'est pas un mode de gouvernement pacifié des grands nombres, elle est "sauvage " selon l'expression de Claude Lefort ${ }^{8}$ ou "l insurgeante "l selon Miguel Abensour'. La démocratie cherche toujours à déborder du cadre. Elle ne peut être qu'une perspective, un horizon, une ligne de conduite. Elle s'institue et se maintient dans la $\|$ dissolution des repères de la certitude $\|{ }^{10}$.

Par-delà les pseudo-consensus, on peut sans doute rappeler que le mot "démocratie" est d'abord une insulte ${ }^{11}$, un terme inventé par ceux qui y sont opposés ${ }^{12}$. Cette haine de la démocratie, haine de "I'innommable gouvernement de la multitude $"$, trouve des échos contemporains dans la dénonciation de "l la société démocratique qui veut que tous soient égaux et toutes les différences respectées $\|^{13}$.

La question du droit de vote des étrangers, question posée dans le cadre des régimes démocratiques modernes, participe du conflit

4. Voir, par exemple, BOIREAU-ROUILLÉ, Monique, "La réduction libérale de la démocratie", in : Critique de la politique, colloque international en hommage à Miguel Abensour, UNESCO, 1820 novembre 2004.

5. Cf. ABENSOUR, Miguel, La démocratie contre l'État : Marx et le moment machiavélien, Paris : Presses universitaires de France, 1997, 115 p. (voir p. 110).

6. On se réfère ici principalement aux travaux (dans leur diversité et parfois leurs divergences) de Miguel Abensour, Giorgio Agamben, Hannah Arendt, Alain Badiou, Étienne Balibar, Marie-Claire Caloz-Tschopp, Cornelius Castoriadis, Monique Chemillier-Gendreau, Jacques Derrida, Claude Lefort, Gérard Mairet, Jean-Luc Nancy, Antonio Negri, Jacques Rancière et Étienne Tassin.

7. Ou, selon les auteurs, division, mésentente, différend...

8. Cf. LEFORT, Claude, Éléments d'une critique de la bureaucratie, Paris : Éd. Gallimard, 1979, 392 p. (voir p. 23).

9. Cf. ABENSOUR, Miguel, La démocratie contre l'État, Paris : Éd. Le Félin-Kiron, 2004 (nouvelle édition), $189 \mathrm{p}$.

10. LEFORT, Claude, Essais sur le politique : XIX゚-XXe siècles, Paris : Éd. du Seuil, 1986, 331 p. (cf. p. 29).

11. Cf. RANCIÈRE, Jacques, La haine de la démocratie, Paris : Éd. La Fabrique, 2005, 106 p. (voir p. 7).

12. Cf. RANCIĖRE, Jacques, Aux bords du politique, Paris : Éd. Gallimard (1 $1^{\text {re }}$ édition 1998), 2004, 261 p. (voir p. 232).

13. RANCIÈRE, Jacques, La haine de la démocratie, op. cit., p. 10. 
proprement politique. II s'agit d'un litige sur la légitimité de la participation politique de certaines catégories de personnes. Et par làmême, il s'agit d'un litige sur les règles politiques de toute la collectivité, voire sur l'existence de la politique elle-même.

Afin de construire une approche théorique du problème du droit de vote des étrangers, on se référera principalement dans les lignes qui suivent aux travaux de Jacques Rancière ${ }^{14}$. Bien que ne portant pas directement sur ce problème ni même sur les notions plus générales de citoyenneté et de nationalité, les thèses de J. Rancière retiennent la dimension essentiellement agonistique de la politique et montrent bien comment, si la démocratie ne peut nullement être réduite au droit de vote, celui-ci constitue une voie d'accès à la vie politique pour les individus et les groupes tenus à l'écart et représente donc une revendication essentielle de toute perspective démocratique.

\section{- Droit de vote et démocratie : une relation nécessaire mais pas évidente}

La relation entre droit de vote et démocratie ne va pas de soi. Le problème ne se pose pas seulement en termes d'affrontement entre démocratie représentative et démocratie participative ${ }^{15}$. La question du droit de vote des étrangers se pose en tant que modalité d'accès à la sphère publique dans son ensemble.

Dans une première approche, le suffrage universel semble aujourd'hui le principal indicateur du régime démocratique ${ }^{16}$. Le droit de vote apparaît comme un attribut fondamental, à la fois symbolique et pratique, de la citoyenneté par laquelle un individu participe à l'exercice du "pouvoir du peuple". Face au discours rationaliste-contractualiste (restreignant le politique à des règles institutionnelles formelles) et à sa critique marxiste (dénonçant le caractère factice de l'égalité formelle entre citoyens abstraits, masquant les inégalités réelles entre les hommes concrets), il est nécessaire de comprendre profondément le rôle joué par le suffrage, à partir du politique, c'est-à-dire à partir d'une conception proprement politique du politique.

14. On se réfère ici en particulier à trois ouvrages : RANCIÈRE, Jacques, La mésentente, Paris : Éd. Galilée, 1995, 187 p. ; RANCIÈRE, Jacques, Aux bords du politique, op. cit. ; RANCIĖRE, Jacques, La haine de la démocratie, op. cit.

15. Voir notamment RANCIÈRE, Jacques, La haine de la démocratie, op. cit., chapitre "Démocratie, République, représentation", pp. 58-78.

16. Cf. ROSANVALLON, Pierre, Le sacre du citoyen : histoire du suffrage universel en France, Paris : Éd. Gallimard, 1992, 490 p. 
Généalogiquement parlant, la démocratie antique utilisait principalement le tirage au sort, seul dispositif permettant la sélection strictement égalitaire parmi les égaux, en dehors de tout titre à gouverner, et qui a fait l'objet d'un "formidable travail d'oubli $\|^{17}$. Si le tirage au sort est devenu impensable, c'est que s'est imposée comme naturelle l'idée que "l le premier titre sélectionnant ceux qui sont dignes d'occuper le pouvoir [est] le fait de désirer l'exercer $\|{ }^{18}$. Jacques Rancière souligne combien l'élection relève d'une logique oligarchique : II Elle est, de plein droit [...], une représentation des minorités qui ont titre à s'occuper des affaires communes [...]. Et l'élection n'est pas davantage en soi une forme démocratique par laquelle le peuple fait entendre sa voix. Elle est à l'origine l'expression d'un consentement qu'un pouvoir supérieur demande $1{ }^{19}$.

Il ne va donc pas du tout de soi, dans la logique de la démocratie, de prôner le droit de vote. Encore faut-il ajouter que le droit de vote n'est pas seulement le droit de participer aux élections (d'élire des représentants), mais aussi celui d'exercer directement le pouvoir de décision (par exemple lors des référendums).

Plus fondamentalement, dans la démocratie, le suffrage joue un rôle important, notamment sur le plan symbolique, en tant qu'il institue et donne une représentation dans la sphère politique à la division sociale $^{20}$. Claude Lefort élucide la dimension symbolique du suffrage universel comme simulacre de dissolution du corps social à l'œuvre dans le scrutin $^{21}$. La pluralité des hommes se manifeste politiquement de multiples façons, mais le suffrage (y compris en tant qu'il est l'organisation de la compétition pour le pouvoir ${ }^{22}$, qui fournit une issue au conflit, tout en laissant la question ouverte) ${ }^{23}$ est l'institution majeure de la politique.

Jacques Rancière dénonce la tendance propre à tout gouvernement de privatiser l'espace politique en réduisant la sphère publique à son profit et en renvoyant les acteurs non étatiques dans le domaine

\footnotetext{
17. RANCIÈRE, Jacques, La haine de la démocratie, op. cit., p. 49.

18. Ibidem, p. 50.

19. Ibidem, p. 60.

20. Cf. GAUCHET, Marcel ; LEFORT, Claude, "Sur la démocratie : le politique et l'institution du social", Textures, $\mathrm{n}^{\circ}$ 2-3, 1971, pp. 7-79.

21. Cf. LEFORT, Claude, Essais sur le politique, op. cit.

22. Cf. GAUCHET, Marcel ; LEFORT, Claude, "Sur la démocratie", art. cité, pp. 52 et ss.

23. Ibidem, p. 10.
} 
de la vie privée ${ }^{24}$. Or, la politique c'est au contraire le processus par lequel l'on apparaît dans la sphère publique. II est très important de comprendre que le droit de vote est finalement un moyen pour des hommes et des femmes d'apparaître sur la scène politique, de prendre part à la politique et d'agir. Ainsi, si la démocratie ne se limite aucunement au droit de vote, si les moyens d'action politique sont multiples, qui pourrait affirmer que le droit de vote n'a aucune importance politique? Qui pourrait affirmer que le fait de priver telle ou telle catégorie de personnes du droit de vote n'a aucune importance sur l'accès de ces personnes à l'action politique, sur la représentation sur la scène politique? Les étrangers ne sont-ils pas objets du discours politique, sans en être les sujets?

Pour Jacques Rancière, II ce qu'on appelle "démocratie représentative" [...] est une forme mixte : une forme de fonctionnement de l'État, initialement fondée sur le privilège des élites "naturelles" et détournée peu à peu de sa fonction par les luttes démocratiques $11^{25}$, et le suffrage universel est lui-même une forme mixte issue de l'oligarchie et des luttes : " Le suffrage universel n'est en rien une conséquence naturelle de la démocratie. La démocratie n'a pas de conséquence naturelle précisément parce qu'elle est la division de la "nature", le lien rompu entre propriétés naturelles et formes de gouvernement. Le suffrage universel est une forme mixte, née de l'oligarchie, détournée par le combat démocratique et perpétuellement reconquise par l'oligarchie qui propose ses candidats et quelquefois ses décisions au choix du corps électoral sans jamais pouvoir exclure le risque que le corps électoral se comporte comme une population de tirage au sort $1{ }^{26}$.

Ces propos situent bien la question du vote dans l'affrontement entre police et politique ${ }^{27}$. Le vote ne relève pas simplement d'une logique de domination comme certains courants critiques l'entendent ${ }^{28}$. Pour comprendre plus profondément l'articulation entre vote et démocratie, il faut voir, au travers de la dualité de l'homme et du citoyen, la question du droit de vote comme objet de revendication, et par là-même comme

\footnotetext{
24. Cf. RANCIÈRE, Jacques, La haine de la démocratie, op. cit., p. 62.

25. Ibidem, p. 61.

26. Ibidem, pp. 61-62.

27. Pour Jacques Rancière, le politique est le lieu d'affrontement entre deux principes, celui de la police (gouvernement, art de la gestion des communautés) et de la politique (mise en actes de la présupposition égalitaire). Voir RANCIĖRE, Jacques, La mésentente, op. cit.

28. Voir, par exemple, BADIOU, Alain, "Considérations philosophiques sur la très singulière coutume du vote, étayées sur l'analyse de récents scrutins en France", Lignes, n 9, novembre 2002, pp. 30-31.
} 
instrument de la conquête démocratique par les sujets politiques. Le pouvoir du peuple, éminemment problématique, réside à la fois II en deçà " des formes juridico-politiques, et " au-delà $\|^{29}$. En effet, le pouvoir du peuple est en dernière instance le fondement du gouvernement démocratique. Et au-delà, la mécanique de police tend toujours à récupérer son pouvoir en détournant les formes démocratiques. Face aux prétentions des gouvernements à réduire la sphère publique et à limiter l'intervention des acteurs non étatiques, la démocratie est le processus de lutte contre cette privatisation et pour l'élargissement de la sphère publique. La lutte pour le droit de vote des étrangers s'inscrit dans cette perspective.

\section{- La lutte pour le droit de vote des étrangers : une lutte pour la démocratie}

La lutte pour l'universalisation du suffrage, et plus globalement pour la reconnaissance de la qualité d'égaux et de sujets politiques à ceux qui en sont privés, est, avec celle contre la privatisation de la sphère publique, une des deux grandes lignes du combat que signifie la démocratie.

Ayant à l'esprit la question de l'extension du droit de vote aux étrangers, on peut se reconnaître dans ces lignes où Jacques Rancière situe les luttes historiques pour l'égalité politique dans leur dimension perpétuellement fondatrice de la démocratie comme mouvement : " Cela a d'abord signifié les luttes pour inclure au nombre des électeurs et des éligibles tous ceux que la logique policière en excluait naturellement : tous ceux qui n'ont pas de titre à participer à la vie publique, parce qu'ils n'appartiennent pas à la "société", mais seulement à la vie domestique et reproductrice, parce que leur travail appartient à un maître ou à un époux : travailleurs salariés longtemps assimilés à des domestiques dépendant de leurs maîtres et incapables d'une volonté propre, femmes soumises à la volonté de leurs époux et commises aux soucis de la famille et de la vie domestique $\|^{30}$. Et plus loin : " Le mouvement démocratique est [...] un double mouvement de transgression des limites, un mouvement pour étendre l'égalité de l'homme public à d'autres domaines de la vie

29. RANCIÈRE, Jacques, La haine de la démocratie, op. cit., p. 62.

30. Ibidem, p. 63. 
commune, et [...] un mouvement aussi pour réaffirmer l'appartenance à tous et à n'importe qui de cette sphère publique incessamment privatisée ${ }^{31}$.

Revenant sur "la dualité tant commentée de l'homme et du citoyen " ${ }^{32}$, Jacques Rancière montre que c'est l'intervalle ouvert entre les catégories (homme et citoyen) qui permet d'ouvrir un espace pour l'action politique : « Des sujets politiques existent dans l'intervalle entre différents noms de sujets. Homme et citoyen sont de tels noms, des noms du commun dont l'extension et la compréhension sont également litigieuses et qui, pour cette raison, se prêtent à une supplémentation politique, à un exercice qui vérifie à quels sujets ces noms s'appliquent et de quelle puissance ils sont porteurs $11^{33}$. C'est effectivement la part de litige que recouvrent ces catégories qui ouvre la voie, historiquement, aux luttes démocratiques, qui permettent aux sujets politiques de se constituer comme tels. La dualité de l'homme et du citoyen permet le jeu combiné sur les deux noms : " Comme nom politique, le citoyen oppose la règle de l'égalité fixée par la loi et par son principe aux inégalités caractérisant les "hommes", c'est-à-dire les individus privés, soumis aux pouvoirs de la naissance et de la richesse. Et à l'inverse, la référence à l' "homme" oppose l'égale capacité de tous à toutes les privatisations de la citoyenneté : celles qui excluent de la citoyenneté telle ou telle partie de la population ou celles qui excluent tel ou tel domaine de la vie collective du règne de l'égalité citoyenne. Chacun de ces termes joue alors polémiquement le rôle de l'universel qui s'oppose au particulier. Et l'opposition de la "vie nue" à l'existence politique est elle-même politisable $1{ }^{34}$. Jacques Rancière illustre son propos en rappelant l'argument paradoxal de la lutte pour la cause des femmes : " La femme a le droit de monter sur l'échafaud; elle doit avoir également celui de monter à la Tribune $1{ }^{35}$, argument qui montre que c'est bien parce qu'elles s'engagent comme citoyennes que les femmes risquent l'échafaud, et donc, c'est qu'elles II exercent, par leur action, le droit des citoyen(ne)s que la loi leur refuse $1{ }^{36}$.

La politique est ainsi une opération de dédoublement, par laquelle des sujets affirment avoir ce qu'ils n'ont pas, et ce faisant, montrent qu'ils l'ont. Ou alors, ils affirment être ce qu'ils ne sont pas, et par ce

\footnotetext{
31. Ibidem, p. 65.

32. Ibidem.

33. Ibidem, p. 66.

34. Ibidem, p. 67.

35. Ibidem.

36. Ibidem, p. 68.
} 
geste, le deviennent. Jacques Rancière rappelle la lutte des Noirs américains pour l'égalité des droits, qui par leur combat montrent qu'ils sont des sujets politiques alors que ce "statut" leur est dénié. Dans le débat sur le droit de vote des étrangers, on pourrait faire le parallèle avec l'argument des impôts, que les étrangers paient, ce qui prouve leur inscription dans la cité, leur contribution en tant que citoyens, qu'ils ne sont pas formellement. De la même façon, on entend encore aujourd'hui l'argument de la dette du sang, qui rappelle la participation des colonisés à l'effort de guerre de la France. S'ils ont été mobilisés pour défendre la France, c'est bien parce qu'ils étaient français, alors qu'ils ne l'étaient pas (ou en tout cas, pas pleinement) ${ }^{37}$. De nombreux couples de catégories sont ainsi des ressources pour la lutte en tant qu'ils ouvrent des intervalles pour la discussion : citoyenneté/nationalité, droit de vote au niveau local/droit de vote au niveau national, communautaires/extracommunautaires, etc.

C'est ainsi que le processus démocratique ouvre sans cesse la voie à des recompositions, à des reconfigurations du privé et du public, de l'universel et du particulier. La démocratie implique donc une "I récusation de la prétention des gouvernements à incarner un principe un de la vie publique et à circonscrire par là la compréhension et l'extension de cette vie publique $11^{38}$.

La revendication du droit de vote des étrangers s'inscrit dans le mouvement perpétuel de l'universalisation du suffrage, affirmant l'égalité comme condition première de la vie en commun, et comme à chaque moment du litige sur l'extension de la citoyenneté à des catégories préalablement exclues, elle est l'émergence de sujets politiques en tant que tels, et elle relève donc pleinement de la démocratie comme mouvement.

\section{- La lutte pour l'égalité : un processus de subjectivation politique}

La revendication du droit de vote des étrangers est aussi politique dans le sens où elle s'inscrit dans un processus d'émancipation, c'està-dire de II vérification de l'égalité de n'importe quel être parlant avec n'importe quel autre $1{ }^{39}$. Comme dans les cas évoqués par Jacques Rancière (travailleurs, femmes, Noirs ou autres), la revendication est

37. Sur ce point, voir ANDRÈS, Hervé, Le droit de vote des étrangers, op. cit.

38. RANCIÈRE, Jacques, La haine de la démocratie, op. cit., p. 70.

39. RANCIÈRE, Jacques, Aux bords du politique, op. cit., p. 115. 
émise "I au nom d'une catégorie [en l'occurrence ici, les étrangers] à laquelle on dénie le principe de cette égalité II. L'égalité, rappelons-le, "I n'est pas une valeur [...], mais un universel qui doit être présupposé, vérifié et démontré en chaque cas [...], elle est un opérateur de démonstrations II. Comme dit J. Rancière, "le mode d'efficacité de l'universalité en politique, c'est la construction, discursive et pratique, d'une vérification polémique, un cas, une démonstration [...]. L'universalité ne réside pas dans les concepts invoqués ['humanité, ses droits], mais dans le processus argumentatif qui démontre leurs conséquences, qui dit ce qu'il résulte du fait que l'ouvrier est un citoyen, le Noir un être humain, etc. [...]. Le schéma logique de la protestation sociale en général peut se résumer ainsi : est-ce que nous appartenons ou non à telle catégorie - citoyens, hommes, etc. - et qu'est-ce qu'il en résulte? L'universalité politique n'est pas dans homme ou dans citoyen. Elle est dans le "qu'est-ce qui en résulte?", dans sa mise en œuvre discursive et pratique $1{ }^{40}$.

En l'occurrence, la volonté que soit accordée aux étrangers l'égalité des droits politiques s'inscrit pleinement dans cette perspective politique d'émancipation. L'argumentation selon laquelle les étrangers sont ici depuis longtemps, ont construit les villes, paient des impôts, contribuent ainsi à la "vie de la cité", consiste à démontrer que les étrangers en question sont déjà des citoyens, auxquels ce statut est dénié. Les slogans des campagnes pour le vote des étrangers - par exemple : "Tous résidents, tous citoyens, tous égaux ", "Un-e habitant-e, une voix ", "Parisiens d'ailleurs, citoyens d'ici ", "Même sol : mêmes droits, même voix "I, " Un(e) résident(e), une voix ", " J'y suis, i'y vote "... - tendent tous à réaffirmer l'inscription dans la cité, qui présuppose l'égalité et que la restriction des droits est une sorte de bannissement ou d'ostracisme insupportable, qui porte atteinte à l'ensemble de la communauté, qui en mine les fondements. Si la plupart de ceux qui militent depuis des années pour le droit de vote des étrangers ne sont pas forcément eux-mêmes des étrangers, ils s'estiment directement concernés, au nom des principes fondateurs de la communauté politique.

Pour Jacques Rancière, "la construction de ces cas d'égalité n'est pas l'œuvre d'une identité en acte ", mais elle est " un processus de subjectivation ", c'est-à-dire "l la formation d'un un qui n'est pas un soi mais la relation d'un soi à un autre $1{ }^{41}$. En l'occurrence, c'est une nouvelle figure du citoyen qui est dessinée par les partisans du droit de vote

40. Ibidem, pp. 116-117.

41. Ibidem, p.118.

Vol. $19, n^{\circ} 114$

novembre - décembre 2007 
des étrangers. Face au discours de police qui objecte que ne sont citoyens que ceux qui ont fait allégeance pleine et entière en acquérant la nationalité, ils affirment la primauté de l'inscription dans le social, via la notion de résidence, comme condition première de l'existence politique. En d'autres termes, ils affirment "Nous sommes tous citoyens " en sachant bien que ce statut leur est dénié collectivement et individuellement, dans sa dimension subversive, car la police exige pour l'octroi de ce statut un réquisit de normalisation qu'ils contestent. Au fond, même si beaucoup sont déjà des Français et donc des citoyens, ils affirment "Nous sommes tous des étrangers "), comme on a pu dire auparavant "Nous sommes tous des juifs allemands " ${ }^{42}$ (et plus récemment " première, deuxième, troisième génération, nous sommes tous des enfants d'immigrés (1), c'est-à-dire qu'ils affirment être ce qu'ils ne sont pas, pour dire qu'ils ne sont pas non plus ce que la police leur dit qu'ils sont. Ils affirment ainsi rejeter la définition du citoyen qu'on leur impose, et qui les concerne directement, basée sur l'allégeance, l'uniformisation et la normalisation que représente la nationalité.

En fait, au travers de la revendication du droit de vote des étrangers, comme l'explique J. Rancière, s'affirme une logique de la subjectivation politique qui marque aussi une triple détermination de l'altérité. Premièrement, elle n'est pas " l'affirmation d'une simple identité ", mais aussi "le déni d'une identité imposée par un autre, fixée par la logique policière $\|$. Elle rejette la conception du national qui est à l'œuvre dans le refus du vote des étrangers. Deuxièmement, elle est une "I démonstration " vis-à-vis de celui qui refuse (l'État-nation), ce qui crée un lieu commun pour la polémique sur l'égalité. Et troisièmement, elle comporte une part d'identification quelque part impossible ${ }^{43}$ ("Nous sommes tous des étrangers 11$)$.

Ainsi, la démocratie est l'enjeu fondamental de la question du vote des étrangers. Dans la continuité des luttes pour l'universalisation du suffrage, le combat pour le droit de vote des étrangers s'inscrit dans une perspective démocratique radicale, en tant que redéfinition des modalités d'accès à la vie politique. Le droit de vote des étrangers apparaît comme le moyen de réaffirmer une conception substantielle de la démocratie, par-delà les débats sur la représentation ou la participation directe. II est un enjeu direct, non seulement pour les personnes concernées (les étrangers), mais pour tous les citoyens.

42. Ibidem, p. 120.

43. Ibidem, p. 121. 


\section{Le droit de vote des étrangers comme nœud de contra- diction entre démocratie et souveraineté}

La question du droit de vote des étrangers, c'est celle de la communauté politique. Par-delà les aspects formels, instrumentaux ou symboliques, l'accès au droit de vote constitue la voie vers la pleine participation à la vie politique, l'appartenance à la communauté politique. Cette question ne porte pas seulement sur le tracé des frontières de cette communauté, mais elle porte, structurellement, sur la définition même de cette communauté. Ce qui nous intéresse particulièrement, c'est la dimension du conflit, qui marque l'opposition entre deux logiques. " De quel droit " ${ }^{44}$ l'étranger prendrait-il part à ce à quoi il n'a pas part? Dans les régimes démocratiques modernes, l'exclusion des étrangers du droit de vote correspond à un certain tracé de la communauté politique, la nationalité, basée sur le principe de souveraineté. Mais de l'autre côté, la revendication du droit de vote des étrangers et le mouvement vers cette ouverture relèvent d'une autre conception de la communauté politique, de la citoyenneté comme principe d'action, et c'est la direction logique de la démocratie comme mouvement. C'est ainsi que le droit de vote des étrangers apparaît comme un point nodal de contradiction dans les fondements du régime, entre nationalité et citoyenneté, ou entre souveraineté et démocratie.

\section{- Le conflit sur le droit de vote des étrangers : un conflit sur la communauté politique}

La question de la communauté politique est essentielle dans le cadre d'une réflexion sur le politique. Aborder la communauté politique par la question des frontières de celle-ci est impossible sans réflexion sur la notion même, sur ce que signifie "communauté politique"

La notion de "communauté" est une des plus redoutables, à cause des utilisations qui peuvent être (et sont) faites du terme. Nous n'ignorons

44. On reprend ici volontairement la formule de Danièle Lochak, montrant combien la présence de l'étranger est toujours conçue comme illégitime. Voir LOCHAK, Danièle, Étrangers : de quel droit ?, Paris : Presses universitaires de France, 1985, $256 \mathrm{p}$

45. Parmi les nombreuses réflexions sur le sujet, on pourrait citer les contributions d'Étienne Tassin (TASSIN, Étienne, Un monde commun. Pour une cosmo-politique des conflits, Paris : Éd. du Seuil, 2003, 311 p.) et les discussions d'Étienne Balibar partant d'une analyse critique de Dominique Schnapper (qui situe sa "communauté des citoyens" comme moyen terme entre Jürgen Habermas et Carl Schmitt), intégrant les déconstructions de la notion de communauté chez Jean-Luc Nancy et Jacques Rancière. Voir BALIBAR, Étienne, "Une citoyenneté sans communauté ?", in: BALIBAR, Étienne, Nous, citoyens d'Europe ? Les frontières, l'État, la démocratie, Paris : Éd. La Découverte, 2001, pp. 93-126. 
pas les dangers d'une conception essentialiste, raciste, qui cherche à figer les individus dans une appartenance et une seule, de manière totalitaire $^{46}$.

À l'instar d'Étienne Tassin, dans son travail à partir d'Hannah Arendt, on conçoit la notion de "communauté politique", en tant que, justement, elle se distingue de toute autre communauté (culturelle, naturelle, économique, religieuse et y compris nationale) par le type de lien qui est tissé entre ses membres. Parler de "communauté politique", ce n'est pas adopter un découpage naturalisant des populations, c'est se référer à l'action politique, c'est-à-dire aux acteurs qui, dans leur pluralité, agissent politiquement, et non pas à la simple agrégation d'individus vivant côte à côte. L'agir est le mode sous lequel le politique se déploie. Le lien politique est le lien tissé par les citoyens engagés dans l'action politique ${ }^{47}$.

Dire que les étrangers sont exclus de la communauté politique, c'est dire quelque chose de fondamental sur la communauté politique et, plus que cela, c'est la construire d'une certaine façon. C'est la logique de la souveraineté. Dire que les étrangers en font partie, c'est dire autre chose de la communauté politique, et c'est la construire d'une autre façon. C'est la logique de la démocratie.

\section{- La nationalité ou la logique de souveraineté}

Le paradigme selon lequel l'État souverain démocratique moderne se caractérise par l'exclusion des étrangers des droits politiques est, à la fois, passablement ébranlé dans les faits et totalement en vigueur dans les esprits. Il s'agit ici de proposer une interprétation sur ce qui bloque non dans les faits, non dans les pratiques, mais dans les fondements théoriques du régime.

Le principe est simple à énoncer : les étrangers n'ont pas le droit de vote, puisqu'ils sont étrangers. Le droit de vote est une des marques par lesquelles l'État souverain trace la frontière entre ses ressortissants et les autres. La nationalité, "clôture sociale " ${ }^{48}$, est ce qui permet d'inclure et d'exclure, de séparer les étrangers et les nationaux, et le droit de vote est un des attributs incontestables de la nationalité. L'étranger, éternel exclu, apparaît dans le système des États souverains

\footnotetext{
46. Voir notamment GAUCHET, Marcel ; LEFORT, Claude, "Sur la démocratie", art. cité, note 3, p. 9.

47. Cf. TASSIN, Étienne, Un monde commun, op. cit., p. 13.

48. BRUBAKER, Rogers, Citoyenneté et nationalité en France et en Allemagne, Paris : Éd. Belin, 1997, 319 p.
} 
comme privé des droits politiques en général et du droit de vote en particulier $^{49}$. La règle générale est donc évidente : les étrangers n'ont pas le droit de vote ${ }^{50}$. Cette règle semble aller de soi pour les esprits du début du XXle siècle, et il convient de noter qu'elle rend compte d'une part de réalité incontestable. Du point de vue du droit de vote, aucun État du monde ne semble traiter sur un plan de stricte égalité ses ressortissants et les étrangers ${ }^{51}$.

Néanmoins, une étude de la situation réelle, du droit positif en la matière, montre que dans au moins un pays sur trois dans le monde des étrangers ont le droit de vote, au moins à certains scrutins ${ }^{52}$. Cette situation est peu connue, et encore moins prise en compte dans les débats sur la question. La pratique du droit de vote des étrangers est, finalement, assez banalisée. Pourtant, elle reste, d'un certain point de vue, impensable, littéralement, ou en tout cas pensable uniquement dans le registre de l'exceptionnalité. Ainsi, l'instauration du droit de vote des citoyens de l'Union européenne (aux scrutins municipaux et européens, dans les pays de l'Union où ils résident et dont ils n'ont pas la nationalité) est vue comme une sorte de transfert, de transmutation du modèle souverainiste, et ce n'est qu'exceptionnellement que le droit de vote relevant de l'État pourrait être copartagé, dans le cadre métaétatique européen, par des normes de réciprocité strictement édifiées.

Ainsi, on ne tire pas les conclusions de la disjonction entre citoyenneté et nationalité, dans le cadre de l'UE. Cette disjonction est réelle, malgré son caractère limité et fragile ${ }^{53}$. Cette disjonction prouve, au niveau de chaque État, qu'il est possible de concevoir et de mettre en œuvre concrètement un droit de vote reposant sur d'autres critères que celui de la nationalité. Si la nouvelle norme à prendre en compte est celle de la réciprocité, alors il conviendrait pour le moins de tirer les conséquences de l'état actuel du droit de vote des étrangers dans certains pays (en accordant le droit de vote aux ressortissants de ces pays),

49. Voir LOCHAK, Danièle, Étrangers : de quel droit ?, Paris : Presses universitaires de France, 1985, chapitre "Les stigmates de l'infériorité", partie "Homo politicus", pp. 130-136.

50. C'est la règle générale, et les entorses à cette règle ne paraissent en première analyse qu'exceptionnelles. Voir DELPÉRÉE, Francis, Les droits politiques des étrangers, Paris: Presses universitaires de France, 1995, collection "Que sais-je ?", 127 p.

51. La Nouvelle-Zélande, le Chili et I'Uruguay accordent, a priori, le droit de vote aux étrangers pour tous les scrutins, mais au bout d'une durée de résidence assez longue. Voir ANDRÈS, Hervé, Le droit de vote des étrangers, op. cit.

52. Voir à ce sujet notre contribution dans ce même numéro, p. 65, et plus complètement ANDRÈS, Hervé, Le droit de vote des étrangers, op. cit.

53. Cf. WIHTOL de WENDEN, Catherine, La citoyenneté européenne, Paris : Presses de la Fondation nationale des sciences politiques, 1997, $105 \mathrm{p}$. 
et il conviendrait également d'établir quelle politique de réciprocité on entend mener, ne pouvant logiquement attendre passivement que tel ou tel État "bouge" avant de "bouger" soi-même. Peut-on réellement faire dépendre les droits des étrangers en France des avancées de la démocratie dans tel ou tel pays? De même, les autres expériences, européennes et extra-européennes, du droit de vote des étrangers alimentent également l'émergence de solutions échappant au modèle classique, à la règle générale. Ces exceptions (les expériences du droit de vote des étrangers) ne font-elles que confirmer la règle? Quand les exceptions se multiplient et prennent une ampleur telle qu'un pays sur trois dans le monde accorde, d'une manière ou d'une autre, le droit de vote à des étrangers, la règle est-elle réellement confirmée? De plus, quand les fondements juridiques de l'exclusion politique des étrangers apparaissent comme purement formels et inconsistants sur le fond ${ }^{54}$, quand la question du vote des étrangers est débattue dans de nombreux pays, on peut être conduit à penser plutôt que c'est la règle générale qui est ébranlée, ou en tout cas remise en question.

La question de fond est politique. II s'agit de savoir si les étrangers font partie de la communauté politique, ou non. La logique de la souveraineté conduit à répondre de la façon suivante : c'est la nationalité qui définit la communauté politique. Peu importent, au fond, les controverses sur telle ou telle conception de la nation, plus ou moins "ethnique" ou plus ou moins "civique" 55 . La nationalité, par-delà les confusions ethnoculturelles, est d'abord un statut juridique liant les individus et un État, un ensemble de droits et de devoirs ${ }^{56}$. Elle est d'abord une relation d'allégeance, car elle est imposée par l'État, et principalement fondée sur l'arbitraire de la naissance. Les règles d'attribution ou d'acquisition de la nationalité, qu'elles soient plus ou moins "ouvertes" ou "fermées", relèvent avant tout du hasard, que ce soit le lieu de sa propre naissance ou celui de ses parents, ou encore la nationalité des parents. La nationalité, c'est le contraire de la liberté. Se présentant comme la modalité de participation à la "souveraineté du peuple", elle masque mal que la délimitation de ce "peuple" est préalablement définie par l'État, niant ainsi la prétendue souveraineté populaire. Si le peuple est souverain, pourquoi est-il amputé d'une partie de lui-même? Pourquoi considère-t-on tels ou tels comme

\footnotetext{
54. Voir ANDRÈS, Hervé, Le droit de vote des étrangers, op. cit., partie juridique, pp. 41-197.

55. Cf. SCHNAPPER, Dominique, La communauté des citoyens : sur l'idée moderne de nation, Paris : Éd. Gallimard, 2003 ( $1^{\text {re }}$ édition 1994), 320 p.

56. Cf. LAGARDE, Paul, La nationalité française, Paris : Éd. Dalloz, 1997 ( $3^{e}$ édition), 374 p.
} 
étrangers? C'est bien l'État qui définit qui sont ses ressortissants et qui sont les étrangers. Selon cette logique, c'est bien la souveraineté qui prime, mais celle de l'État.

Dans les faits, si l'émergence des États souverains modernes a correspondu à un certain développement de la démocratie politique et sociale, dans certains endroits de la planète l'enfermement "nationalitaire" aboutit aujourd'hui à une impasse, car la souveraineté semble ne subsister que dans ses dimensions répressives, les États souverains ayant renoncé à protéger leurs populations des ravages de la globalisation économique ${ }^{57}$. Exclure les étrangers de la participation politique, ou exiger d'eux qu'ils se soumettent à une procédure spéciale, la "naturalisation", c'est-à-dire, littéralement, une conversion de l'être humain dans sa propre nature, c'est concevoir la communauté politique comme préalablement donnée, essentialisée et, fondamentalement, soumise à la souveraineté de l'État.

Bien que se présentant comme "citoyenneté" des États-nations, la nationalité constitue une dénaturation, voire une négation de la citoyenneté ${ }^{58}$.

\section{- La citoyenneté ou la logique de démocratie}

Proposer, revendiquer le droit de vote des étrangers, c'est concevoir la communauté politique selon la logique de la démocratie, c'est rendre à la citoyenneté son caractère éminemment politique. II s'agit de participation à la politique en tant qu'action. Dit autrement, il s'agit de l'accès à la liberté par la mise en actes de la présupposition égalitaire ${ }^{59}$. Comme concrétisation du projet démocratique, la citoyenneté est un rapport d'égalité au sein de l'agir en commun qui est le propre de l'action politique. Observons que si "I l'en-commun de la cité n'a pas d'autre identité que l'espace où les citoyens se croisent ", et s'il " n'y a pas d'autre unité que l'extériorité de leurs rapports II, la citoyenneté, d'une certaine manière, "I selon son concept est toujours virtuellement "mondiale" 1$)^{60}$. La citoyenneté est fondamentalement universelle. Même

57. Cf. CHEMILLIER-GENDREAU, Monique, Droit international et démocratie mondiale : les raisons d'un échec, Paris : Éd. Textuel, 2002, 270 p.

58. Cf. CHEMILLIER-GENDREAU, Monique, "Quelle citoyenneté universelle adaptée à la pluralité du monde?", in : DAYAN-HERZBRUN, Sonia; TASSIN, Étienne (sous la direction de), Citoyennetés cosmopolitiques, Tumultes, $\mathrm{n}^{\circ}$ 24, mai 2005, pp. 165-178.

59. Cf. RANCIÈRE, Jacques, La mésentente, op. cit.

60. NANCY, Jean-Luc, "Renouer le politique", Intersignes, n 8-9, 1996, pp. 111-126 (cf. p.112). 
si l'organisation politique du monde en États souverains entrave cette universalité, elle est potentiellement déjà présente dans le concept politique de citoyenneté.

Ainsi, la revendication du droit de vote des étrangers porte atteinte à la définition de la citoyenneté "nationalitaire", pas seulement parce qu'elle propose de dissocier citoyenneté et nationalité, mais aussi, et peut-être surtout, parce qu'elle atteste de la citoyenneté déjà manifeste des étrangers qui se lèvent pour réclamer la conquête de ce droit.

Au fond, la différence entre nationalité et citoyenneté ouvre un écart pour la lutte et, par là-même, est une ressource pour la démocratie en actes. Par la volonté d'inclure la part des sans-part dans le compte du demos, ébranlant les fondements de l'ethnos ${ }^{61}$, c'est la démocratie qui s'exprime à nouveau.

C'est ainsi que la citoyenneté (au contraire de la nationalité) n'a que faire du destin, de la naissance ou de la soumission au souverain. Elle est manifestation de liberté, libre choix volontaire, elle est active, revendiquée par des individus qui se dressent pour exiger leur part dans le décompte, pour participer à l'en-commun de la cité.

On pourra sans doute objecter que, définie ainsi, la citoyenneté est pure utopie, et que l'histoire politique (y compris celle de la démocratie moderne) est celle du citoyen portant allégeance au souverain, que ce soit chez Bodin, chez Hobbes ou chez Rousseau. Il est vrai que la Révolution "française" a vite tourné le dos à ses visées universalistes, confrontée à ses limites géographiques et aux menaces des autres États. La confusion entre nationalité et citoyenneté est en partie héritée des évolutions postrévolutionnaires, en passant par le Code civil napoléonien et aboutissant aux textes de la Ille République. Mais l'histoire des révolutions modernes est justement celle de l'irruption des citoyens se dressant contre le monarque. Les citoyens des colonies d'Amérique du Nord proclament leurs droits en coupant tout lien d'allégeance au monarque. La marque distinctive pour cette irruption est celle de la participation à la révolution et non pas la détention du titre de sujet de Sa Majesté. Ce sont les "citoyens" de France qui proclament les droits de l'homme et du citoyen, et si très vite la Constitution institue différents statuts réservant l'action (" citoyenneté active ") à certains, la 
Révolution française est avant toute chose irruption des citoyens dans la scène politique et renversement de l'ordre institué.

On comprend donc que la citoyenneté dans son caractère politique résiste à tout contingentement dans un statut juridique. L'enfermement de la citoyenneté dans la nationalité dénature totalement son concept politique, universaliste et émancipateur.

La proposition du vote des étrangers, en tant qu'elle est une donnée essentielle de la citoyenneté, agit comme le révélateur de la tension existant entre les deux notions de nationalité et de citoyenneté.

Dans cette logique, la notion même de communauté politique rompt avec la vision essentialisée du politique selon laquelle on est ou on n'est pas de la communauté, vision présentée comme naturelle. C'est plutôt l'espace d'exposition en commun, y compris dans sa dimension conflictuelle, qui permet de concevoir la politique comme mode d'action spécifique. Reconnaître aux étrangers le droit de vote, c'est fondamentalement reconnaître l'existence et la participation politiques des personnes qui sont ici, et là, sans allégeance préalable.

Ainsi, le problème du droit de vote des étrangers révèle l'épuisement théorique du modèle démocratique des États souverains. La question qui est posée est celle d'une refondation démocratique, par-delà le principe de souveraineté. La souveraineté a sans doute "épuisé" son potentiel émancipateur ${ }^{62}$, et il convient de reprendre la critique arendtienne : " Là où des hommes veulent être souverains, en tant qu'individus ou que groupes organisés, ils doivent se plier à l'oppression de la volonté, que celle-ci soit la volonté individuelle par laquelle je me contrains moi-même, ou la "volonté générale" d'un groupe organisé. Si les hommes veulent être libres, c'est précisément à la souveraineté qu'ils doivent renoncer $1{ }^{63}$. Ceci implique de remettre en question une conception de la liberté basée sur la volonté, et en particulier de s'engager dans une critique philosophique de la volonté générale comme fondement théorique de la souveraineté populaire ${ }^{64}$.

62. Voir MAIRET, Gérard, Le principe de souveraineté : histoire et fondements du pouvoir moderne, Paris : Éd. Gallimard, 1996, $311 \mathrm{p}$.

63. ARENDT, Hannah, La crise de la culture : huit exercices de pensée politique, Paris : Éd. Gallimard, 1972, 384 p. (cf. p. 214).

64. Cf. TASSIN, Étienne, Un monde commun, op. cit., pp.100 et ss. 